\title{
Ten years of ICESP - Profile of Endometrial Cancer treatment
}

C. Anton, E.V.L. Mayerhoff, R.T. Kleine, J.P. Carvalho

ICESP - Instituto do Câncer do Estado de São Paulo

\section{Objectives}

To describe the characteristics of patients with endometrial cancer treated in a reference cancer center in São Paulo, Brazil.

\section{Methods}

This retrospective study included 703 patients with endometrial cancer diagnosis assisted at the ICESP from 2008 to 2018. Patient data were collected from electronic records. All the diagnoses were based on the anatomopathological study of surgical specimens. Other data analyzed were age at diagnosis, race, body mass index, histologic type and grade, and surgical staging according to the FIGO (2009) criteria. Treatment outcomes were reported according to histological type, surgical FIGO stage, lymph-vascular space involvement and lymph node metastasis.

\section{Results}

Seven hundred and three patients were analyzed according to their clinical, pathological and surgical characteristics (Table 1). The presence of lymphvascular space invasion was associated with a worse overall survival. The median of tumor size was $4 \mathrm{~cm}$. Overall survival in 5 years was $85.3 \%$. Patients with stage II and III had the same overall

survival. Overall survival according to stage, lymph-vascular space invasion and grade can be seen in Figure 1.

\section{Conclusion}

Patients assisted at the ICESP in the last ten years presented with large tumors (4 $\mathrm{cm})$. Lymph-vascular space invasion, histologic grade and stage had an influence on patients' overall survival. Stage II and III patients presented similar overall survival rates in 5 years.
Table 1 Clinical, pathological and surgical characteristics

\begin{tabular}{|c|c|c|}
\hline & & $\mathbb{N}$ \\
\hline \multirow[t]{3}{*}{ Age } & & $63.2(27.9-92.4)$ \\
\hline & $\leq 50$ years & $37(5.396)$ \\
\hline & $>50$ years & $666(94.796)$ \\
\hline BMI & & $31.1(16-58.9)$ \\
\hline \multirow[t]{3}{*}{ Race } & Caucasian & $550(78.296)$ \\
\hline & Black & $131(18.696)$ \\
\hline & Others & $20(2.8 \%)$ \\
\hline \multirow[t]{4}{*}{ Histological type } & Endometrioid & $567(80.796)$ \\
\hline & Serous & $93(13.296)$ \\
\hline & Clear cell & $28(4.096)$ \\
\hline & Others & $15(2.1 \%)$ \\
\hline \multirow[t]{2}{*}{ Grade (endometrioid) } & $\mathrm{G} 1-\mathrm{G} 2$ & $467(82.496)$ \\
\hline & $\mathrm{GB}$ & $99(17.596)$ \\
\hline Tumor size & & $4(0-17)$ \\
\hline \multirow[t]{3}{*}{ LVI } & Present & $280(29.696)$ \\
\hline & Absent & $472(67.15)$ \\
\hline & Unknown & $23(3.396)$ \\
\hline \multirow[t]{9}{*}{ FIGO stage } & IA & $298(42.496)$ \\
\hline & $\| \mathrm{B}$ & $151(21.5 \%)$ \\
\hline & $\|$ & $50(7.196)$ \\
\hline & \|\| $\mathrm{A}$ & $40(5.7 \%)$ \\
\hline & IIIB & $11(1.696)$ \\
\hline & \|\|$\subset 1$ & $49(7.096)$ \\
\hline & $\| 11 \subset 2$ & $59(8.49)$ \\
\hline & IVA & $7(1.096)$ \\
\hline & IVB & $38(5,496)$ \\
\hline \multirow[t]{4}{*}{ Surgery type } & Laparotomy & $182(25.9 \%)$ \\
\hline & Laparoscopic & $480(68.396)$ \\
\hline & Robotic & $43(6.196)$ \\
\hline & Vaginal & $2(0,396)$ \\
\hline
\end{tabular}

*LVI: Lymphovascular invasion

Figure 1: Overall survival related to diferente endometrial cancer parameters

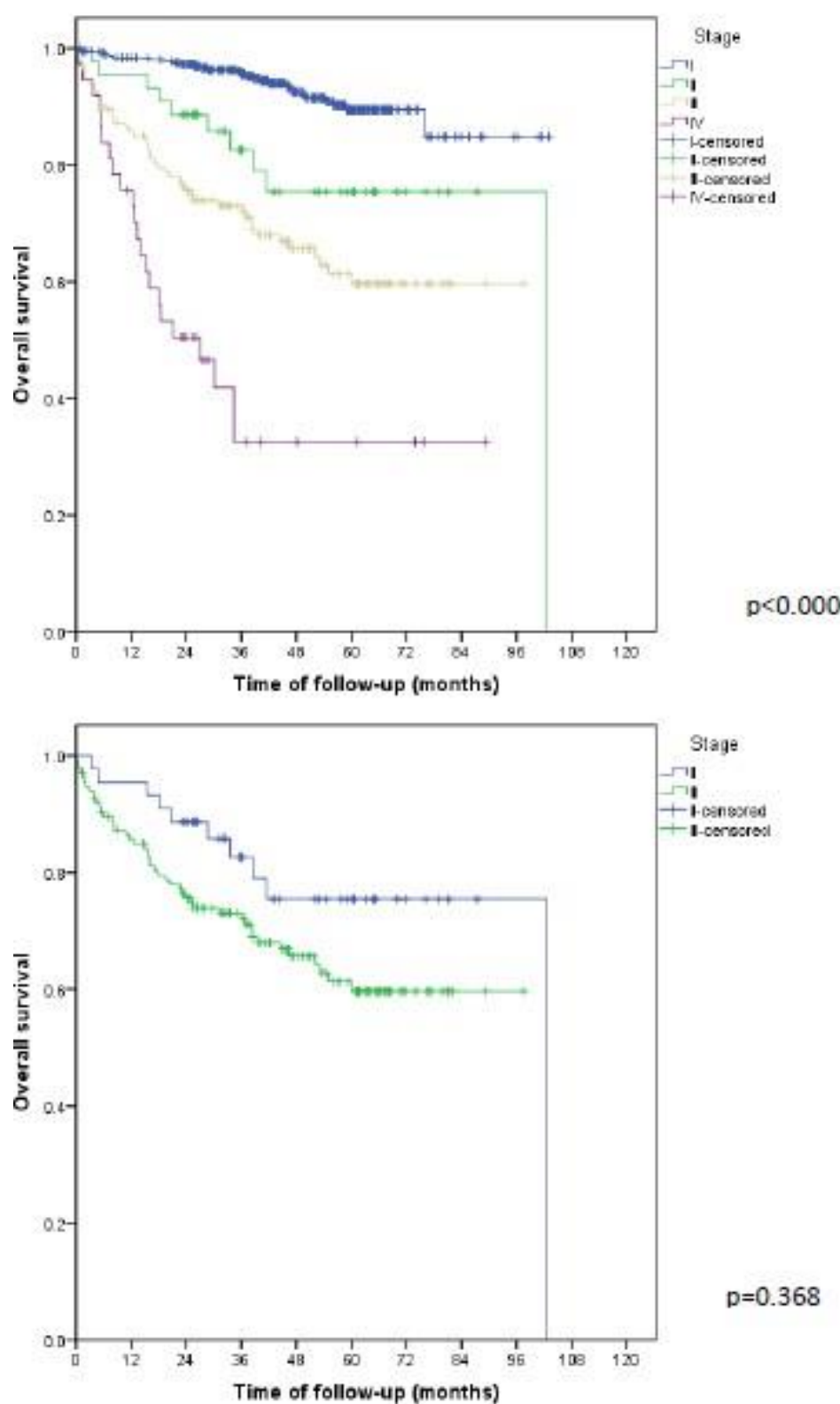

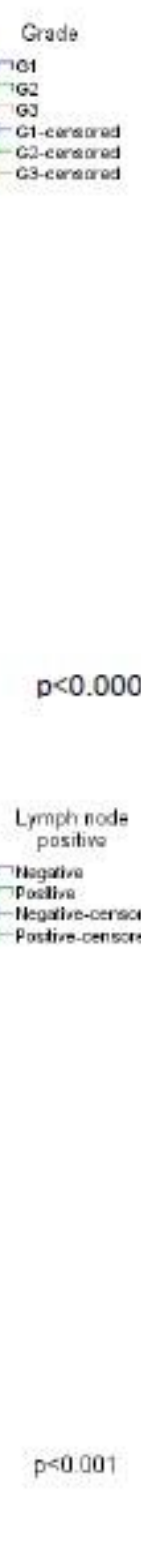

